\title{
No-reference image quality assessment with multi- scale weighted residuals and channel attention mechanism
}

Changzhong Wang ( $\nabla$ changzhongwang@126.com )

Bohai University https://orcid.org/0000-0002-4136-2433

Xiang Lv

Bohai University

Weiping Ding

Nantong University

Xiaodong Fan

Bohai University

\section{Research Article}

Keywords: Multi-scale, No-reference image quality assessment, Channel attention, Active weighted mapping strategy

Posted Date: February 17th, 2022

DOI: https://doi.org/10.21203/rs.3.rs-1185515/v1

License: (c) (i) This work is licensed under a Creative Commons Attribution 4.0 International License. Read Full License 


\title{
No-reference image quality assessment with multi-scale weighted residuals and channel attention mechanism
}

\author{
Changzhong Wang ${ }^{\mathrm{a}, *}$, Xiang $\mathrm{Lv}^{\mathrm{a}}$, Weiping Ding ${ }^{\mathrm{b}}$, Xiaodong, Fan ${ }^{\mathrm{a}}$ \\ ${ }^{a}$ Department of Mathematics, Bohai university, Jinzhou, 121000, P.R. China \\ ${ }^{\mathrm{b}}$ School of Information Science and Technology, Nantong University, Nantong 226019, P.R. China
}

\begin{abstract}
Image is an important information source for human perception and machine pattern recognition. Image quality determines the accuracy and sufficiency of the information obtained. With the rapid development of deep learning in image processing, no-reference image quality assessment (NR-IQA) plays a significant role. Currently, most NR-IQA methods mainly use the global features of images without paying attention to the detail-rich local features and the dependencies between channels. There are subtle differences in detail between distorted and reference images, and the contribution of different channels to image quality assessment differs. Additionally, multi-scale feature extraction can be used to fuse the detailed information of images with different resolutions. The combination of global and local features plays a vital role in extracting image features. Therefore, a multi-scale residual convolutional neural network with an attention mechanism (MsRCANet) is proposed for NR-IQA. First, the network extracts global features and processes local features. Specifically, a multi-scale residual block is used to extract features from distorted images. Then, the residual learning with active weighted mapping strategy and channel attention mechanism is used to further process image features to obtain more abundant high-frequency information. Finally, the fusion strategy and full connection layer are used to evaluate the quality of the proposed network. The experimental results on four synthetic databases and three in-the-wild IQA databases, as well as validation results on cross-database, show that the proposed method has good generalization ability and can be compared with the most advanced methods.
\end{abstract}

Keywords-Multi-scale; No-reference image quality assessment; Channel attention; Active weighted mapping strategy

\section{Introduction}

With the rapid development of computer technology and mobile devices, image information has become essential for perceptual content in daily life and learning. However, in storage devices, the image undergoes compression, copy, transmission, and other operations and will be subjected to various distortions. Therefore, image quality assessment (IQA) has become essential in computer image vision and image processing [1,2].

*Corresponding author. Email address: changzhongwang@126.com; 
Generally, IQA is divided into two categories: subjective and objective evaluations. Subjective evaluation is to evaluate the image subjectively with people as the observer to reflect people's visual perception. Objective evaluation calculates the image quality by designing models and algorithms, reflecting the subjective perception of human eyes, and giving the results based on digital calculation. The objective IQA technology can be divided into three categories: full-reference (FR), reduced-reference (RR), and no-reference (NR). Many IQA methods are not easy to obtain reference images due to their factors or environmental impact on the real practical application. Thus, the FR-IQA and RR-IQA methods are greatly limited in the application of IQA. NR-IQA does not need many reference images, so this technology has attracted significant attention of researchers.

Due to the rapid development of deep learning, convolutional neural network (CNN) has been successfully applied in the field of computer vision [3,4], such as image super-resolution and recognition tasks. It has achieved many remarkable results [5]. Although CNN has shown amazing potential in many visual tasks, NR-IQA remains challenging due to the lack of realrich samples for training. First of all, since there are a large number of parameters in CNNs, the learning of these parameters needs to be conducted under a large number of label data. For NRIQA, the number of reference images in existing synthetic IQA databases, such as LIVE [6], CSIQ [7], TID2008 [8] and TID2013 [36] is relatively small, with only about 20 reference images. For example, the in-the-wild IQA databases LIVE challenge [9], Koniq-10K [10] and SPAQ [38] contain 1,169, 10,073 and 11,125 images with different contents; However, there are no reference images. Second, image distortion is usually reflected in the variation of the details of high-frequency information for the entire image. Generally, the more severe the image distortion, the more the high-frequency information is lost, whereas the low-frequency information almost does not change. If people do not consider the observation process of objects and only think about the problem from the pixel value of images, they will ignore some real details and only pay attention to the dense contour information; thus, increasing the difficulty of learning.

To solve the problem of the small number of training samples, Kang et al. [11] proposed a very shallow network. They proposed an effective method to obtain the image quality of the global region by learning and predicting the image quality of the local region using $32 \times 32$ image blocks as input. This method employed image enhancement technique, which is essential for image denoising or super-resolution problems. However, the network structure is relatively simple, and the number of network layers is relatively shallow. Only one layer of the network structure with a convolution kernel of $7 \times 7$ size is used to extract image features, and local features cannot describe global features. Therefore, this method cannot meet the requirement of sufficient accuracy.

Many researchers have devoted themselves to the task of NR-IQA based on CNNs and proposed many effective algorithms. Li and Dash et al. [12,13] used a full image of 224 x 224 
pixels to train the network. Pan and Zuo et al. [16,17] proposed saliency maps to adjust the weight of each patch. Kim et al. [19] predicted subjective scores by learning human visual perception behaviors. Lin et al. [20] introduced GAN into quality assessment network to provide fake reference images for NR-IQA. Yang et al. [21] proposed an NR-IQA network based on a two-stream CNN structure, which inputs the original and gradient images into the network.

We know that convolution kernels of different sizes can be used to extract different features. Li et al. [54] adopted multi-scale convolution to process image for super-resolution and obtained more effective high-frequency details, thus achieving good results. Inspired by this, Chen et al. [37] also applied multi-scale feature extraction to the field of image quality assessment. Experiments show that multi-scale feature extraction can also improve the performance of quality assessment when processing images. In addition, further fusion of images with different resolutions can alleviate the loss of excessive details in severely distorted images. Channel attention mechanism and active weighted mapping strategy have achieved good results in the super-resolution direction of image processing. They mainly simulate human vision system for weighted image processing. However, as far as we know, no one has combined multi-scale feature extraction, channel attention mechanism and active weighted mapping into IQA.

Therefore, in order to solve the above problems, this study proposed a multi-scale residual CNN with attention mechanism to extract contour features and process detail features and channel correlation. Firstly, the multi-scale feature extraction module used is different from the above [54] and [37]. Multi-scale residual block (MSRB) with three parallel branches is used for feature extraction of distorted images. Multi-scale feature extraction can fuse the detailed information of images with different resolutions. Therefore, when extracting image features, the combination of global features and local features plays an important role. In addition, the network uses residual learning of active weighted mapping strategy (AWMS) and channel attention mechanism (CAM) to further process image features and obtain richer high-frequency information.

The contributions of this study are as follows:

- A novel neural network structure is proposed to learn image quality features better and evaluate image quality more accurately.

- MSRB with three parallel branches is proposed to extract the distorted image. The residual learning with AWMS is used in MSRB. The purpose is to redesign the input mapping instead of directly adding the two results of residual learning.

- CAM is introduced into the IQA task. The corresponding weight values of different channels are deduced through this mechanism. Then, the weight values are assigned to different channels through this mechanism to improve the cross-layer information flow. The remainder of the paper is organized as follows. Section 2 briefly introduces some 
methods related to the proposed network. Section 3 describes the proposed network model. Section 4 describes the experimental details and conducts comparative and cross-validation. Finally, Section 5 presents the conclusion and future studies.

\section{Related works}

\subsection{No-reference Image Quality Assessment Based on CNN}

Several years ago, Kang et al. [11] first introduced CNN into the field of NR-IQA and proposed a network with only five layers, which has crucial research significance. The method adopts the image block-training strategy. The original image is first divided into nonoverlapping $32 \times 32$ image blocks, and each image block's quality is assumed to be roughly the same as that of the original image. Then, the image blocks are successively fed into the network for training parameters. This strategy was made for the lack of samples in the training database, and the proposed network pushed NR-IQA to a high level. Since then, many scholars have devoted themselves to research on applying CNN to NR-IQA. However, the network proposed by Kang et al. has only one convolutional layer, which is a relatively shallow. Thus, the network has some limitations and fails to meet the requirements of accuracy.

Inspired by Kang's idea, Li and Dash et al. [12,13] proposed a CNN with multiple layers of structures. They trained the network using a full image with a size of 224 x 224 pixels. Although the small patch block segmentation technique is not used, relatively good results are obtained on some datasets with fewer reference images due to the deep network structure. Sun et al. [14] and Bosse et al. [15] partially modified the deep CNN network [12,13] by finetuning parameters and achieved remarkable effects. Additionally, Pan et al. [16] and Zuo et al. [17] designed a deep network for small patches. In this network, a significance map detection method based on CNN was proposed. The weight of each patch was adjusted by the significance map, and the quality score of the entire image was output at last. Cheng et al. [18] proposed a NR-IQA network based on a pre-significance map and proved through experiments that the prediction error of significance information was lower than that of a uniform region. Based on this fact, they proposed a pre-SM algorithm that can assign relatively large weights to small blocks of significance graphs to reduce the error.

Recently, Kim et al. [19] proposed a new distorted IQA (DIQA) model. The training of this model is divided into two parts. The first part is to determine the information related to the distorted and reference images. The second part mainly uses human subjective scores to finetune the proposed $\mathrm{CNN}$ network to predict subjective opinion scores by learning human visual perception behavior. However, when the network randomly clips the input image, the patch overlaps will be caused, leading to double calculation and waste time and resources. Lin et al. [20] proposed an NR-IQA network based on a generative adversarial network. In this model, GAN was introduced into the quality assessment network to provide pseudo-reference graphs for NR. Furthermore, the loss of quality perception is added to the generator of GAN, and the 
discriminator is used to discriminate the pseudo-reference graph. Although the effect of the above methods for the NR-IQA has been effectively improved and enhanced, the problem of gradient propagation weakening or disappearing may occur in some databases due to the deep network. To solve this problem, Yang et al. [21] proposed an NR-IQA network based on a twostream CNN structure, which inputs original and gradient images into the network, enabling the network to extract different features representing distortion. Chen et al. [377] applied multiscale feature extraction convolution to the field of image quality evaluation. In the field of computer vision super-resolution, Li et al. [54] first used a multi-scale residual convolution kernel to process images, thereby achieving a good zoom performance effect. Sun and Wang et al.[55] took the structure and texture similarity of the feature map extracted by the intermediate layer as the feature representation of the full-reference model, and took the global average and standard deviation of the final feature map fused by the intermediate feature map as the noreference model feature. Sun et al. [63] proposed a ladder structure to better learn quality perception features. This structure hierarchically integrates the features of the middle layer into the final feature representation, so that the model can make full use of the low-level to highlevel vision information.

\subsection{Different Features Extraction Blocks}

He et al. [22] proposed a residual block (Fig. 1(1)) to avoid the disappearance or explosion of gradient during network training, which can retain the original input features. Some researchers proposed inception blocks (Fig. 1(2)) using convolution kernels of different sizes to extract richer features from images. Zhang and Tian [23] designed a residual dense block (Fig. 2(3)) consisting of a stack of residual and dense blocks. The residual dense block can fully extract local and global features.

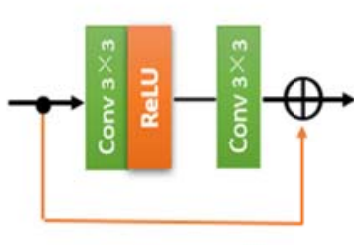

(1) Residual block

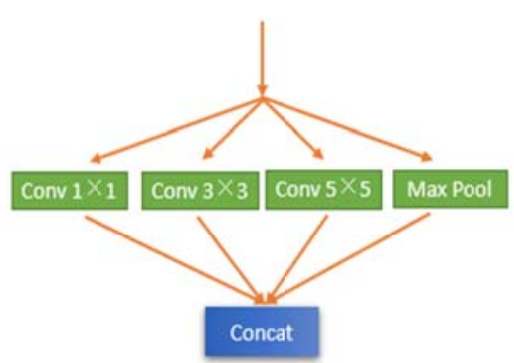

(2) Inception block

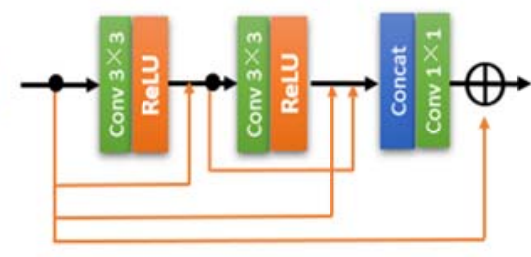

(3) Residual dense block

Fig. 1. Different features extraction blocks

\subsection{Channel Attention}

The unique brain signal processing mechanism of human vision is visual attention. By quickly scanning a global image, we can obtain the important target areas that need attention. 
Then, add more attention to this part to obtain more detailed features and suppress some relatively useless features. The attention mechanism in deep learning draws lessons from human attention thinking mode. Recently, many researchers have devoted themselves to exploring the mechanisms of attention in deep learning. Hu et al. [24] proposed a squeeze and excitation (SE) block, which adaptively adjusts the weight of channels by learning the dependence of each channel. Zhang et al. [25] introduced a deep residual channel attention network (RCAN) with CAM. The network combined residual learning with SE block to process input images and adjusted different features maps according to the degree of dependence between input images. Then, appropriate weights were assigned to these feature maps. The experimental results show that CAM can improve image processing.

\subsection{Active Weighted Mapping}

In general residual learning, the original input information is added to the information obtained after some convolution layers, and the final information is obtained. Fig. 2 (1) shows the simplest residual learning defined as follows:

$$
\mathrm{y}=x+F(x)
$$

where $x$ and $y$ represent the input and output of the residual network, and $F(\cdot)$ is the convolution function of residual learning.

Based on residual learning, Jung et al. [26] proposed an active weighted mapping (AWM) strategy in 2018. AWM mechanism is different from residual learning. It is not the result of adding two paths directly but redefines the mapping of residual learning. Specifically, the AWM mechanism thinks that the weights of $x$ and $F(x)$ are different. After the AWM mechanism, different weights (Fig. 2 (2)) can be derived and redistributed. AWM mechanism can be written as follows:

$$
\mathrm{y}=\lambda x+\mu F(x)
$$

where $\lambda$ and $\mu$ are the weight values calculated by AWM mechanism.

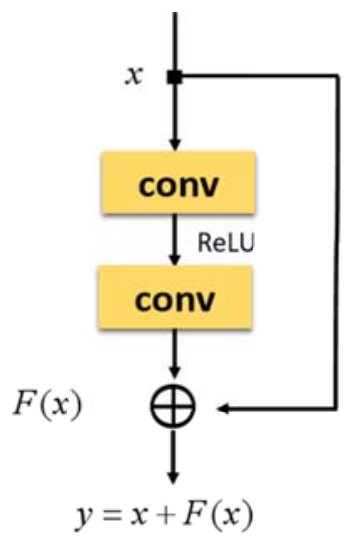

(1)

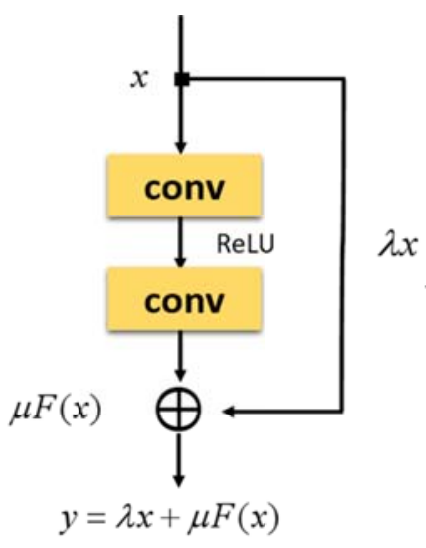

(2)

Fig. 2. General residual learning and Active weighted mapping (AWM) 


\section{Proposed method}

\subsection{A New Multi-Scale Residual Block (MSRB)}

Before the NR-IQA deep network model was proposed, an MSRB structure was first presented (Fig. 3). $S^{n-1}$ and $S^{n}$ are the input and output of MSRB, respectively. This module consists of three parallel branches of different convolution kernels and an AWMS mechanism. The structure of MSRB is described in detail below.

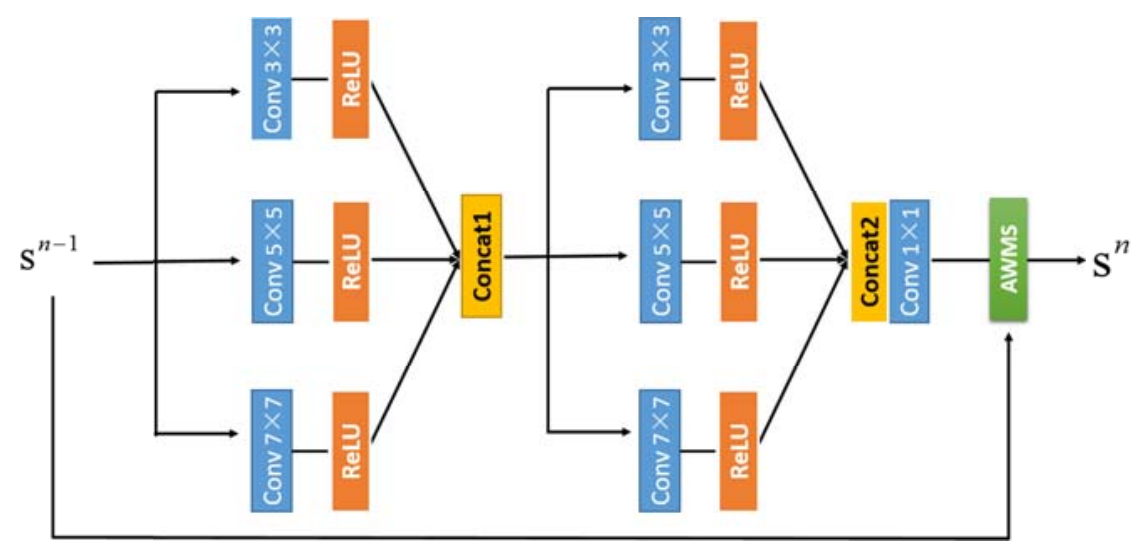

Fig. 3. Multi-scale Residual Block (MSRB)

In the first part, three convolution kernels of different sizes, $3 \times 3,5 \times 5$, and $7 \times 7$, are used to extract multi-scale features from input features. After the convolution operation, the LRelu function is used to obtain richer context information. Then, the three output features are concatenated into a tensor. The process is repeated twice. Finally, the $1 \times 1$ convolution kernel is used to adjust the channel number of output features. This part can be described as follows:

$$
\begin{aligned}
& A_{11}=L\left(\mathrm{~W}_{11}^{3 \times 3} * S^{n-1}+b_{11}^{3 \times 3}\right) \\
& A_{12}=L\left(\mathrm{~W}_{12}^{5 \times 5} * S^{n-1}+b_{12}^{5 \times 5}\right) \\
& A_{13}=L\left(\mathrm{~W}_{13}^{7 \times 7} * S^{n-1}+b_{13}^{7 \times 7}\right) \\
& \mathrm{C}_{1}=\left[A_{11}, A_{12}, A_{13}\right] \\
& B_{21}=L\left(\mathrm{~W}_{21}^{3 \times 3} * C_{1}+b_{21}^{3 \times 3}\right) \\
& B_{22}=L\left(\mathrm{~W}_{22}^{5 \times 5} * C_{1}+b_{22}^{5 \times 5}\right) \\
& B_{23}=L\left(\mathrm{~W}_{23}^{7 \times 7} * C_{1}+b_{23}^{7 \times 7}\right) \\
& \mathrm{C}_{2}=\left[A_{21}, A_{22}, A_{23}\right] \\
& D=f\left(\mathrm{~W}_{3}^{1 \times 1} * \mathrm{C}_{2}+b_{3}^{1 \times 1}\right)
\end{aligned}
$$

where $\mathrm{W}_{i j}^{k \times k}$ and $b_{i j}^{k \times k}$ represent the weight and bias of the $j$-th branch in the $i$-th layer, respectively, using $k \times k$ convolution kernel, $k=3,5,7, i=1,2, j=1,2,3$. $L(\cdot)$ represents the LRelu function, $A_{i j}$ and $B_{i j}$ represent the results obtained through LRelu functions. Concat 
represents the fusion operation, and $D$ represents the result obtained after the $1 \times 1$ convolution kernel.

In the second part, AWMS is used to deal with the output feature maps $D$ and the initial input feature maps $S^{n-1}$ obtained in the first part (see Fig. 4). Suppose $S^{n-1}=\left\{S_{1}, S_{2}, \ldots, S_{k}\right\}$ has $k$ different feature maps, and the size of feature maps is $M \times N$. After global average pooling, the global average channel information $Z=\left\{z_{1}, z_{2}, \ldots, z_{k}\right\}$ is obtained. It means that the twodimensional feature map on each channel will become a statistic after global average pooling, and the $c$-th element of $Z$ can be expressed as follows:

$$
z_{c}=\operatorname{Ave}\left(S_{c}\right)=\frac{1}{M \times N} \sum_{i=1}^{M} \sum_{j=1}^{N} S_{c}(i, j)
$$

where Ave $(\cdot)$ is the global average pool operation, and $S_{c}(i, j)$ is the value of the position $(i, j)$ in the $c$-th channel feature map. As shown in Fig. 4, $Z^{1}$ and $Z^{2}$ represent two statistics after global average pooling.

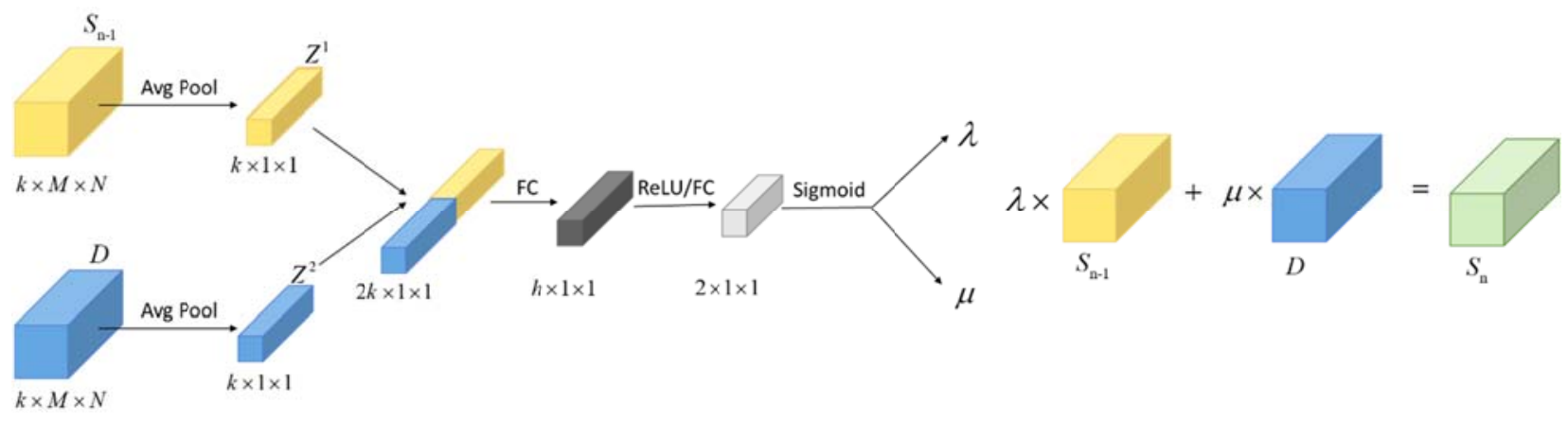

Fig. 4. Active weighted mapping strategy (AWMS)

To obtain the weights of $D$ and $S^{n-1}$, we concatenate $Z^{1}$ and $Z^{2}$ into $Z=\left\{Z^{1}, Z^{2}\right\}$. The weights $\lambda, \mu$ of $S^{n-1}$ and $D$ are calculated using two nonlinear fully connected layers. The process is described as follows:

$$
\begin{aligned}
& Z^{1}=\operatorname{Ave}\left(S^{n-1}\right)=\frac{1}{M \times N} \sum_{i=1}^{M} \sum_{j=1}^{N} S^{n-1}(i, j) \\
& Z^{2}=\operatorname{Ave}(D)=\frac{1}{M \times N} \sum_{i=1}^{M} \sum_{j=1}^{N} D(i, j) \\
& Z=\left[Z^{1}, Z^{2}\right] \\
& \gamma=\sigma\left(\mathrm{W}_{2} \delta\left(\mathrm{W}_{1} Z+b_{1}\right)+b_{2}\right), \gamma=\{\lambda, \mu\} \\
& S^{n}=\lambda S^{n-1}+\mu D
\end{aligned}
$$

where $\mathrm{W}_{1} \in \mathrm{R}^{h \times 2 k}$ and $\mathrm{W}_{2} \in \mathrm{R}^{2 \times h}$ represent the weight matrices of the fully connected layers, $h(2<h<2 k)$ represents the number of dimension nodes of hidden layer, $\sigma$ and $\delta$ represent 
Sigmoid and Relu functions, respectively. $\gamma=\{\lambda, \mu\}$ is the weight vector of $D$ and $S^{n-1}$, and $S^{n}$ is the output of MRSB.

\subsection{Channel Attention Mechanism (CAM)}

In general, it is considered that the feature map of each channel input is equally important. In fact, the importance of feature information input of each channel is different; thus, each channel should be given a different weight. This study uses CAM similar to [24] to give weights to each channel. As shown in Fig. 5, CAM consists of two parts: squeeze and excitation. These two parts are used to learn the weight of each channel to generate the attention of the channel domain. Next, the structure and operation process of squeeze will be given in detail.

\section{Squeeze Excitation}

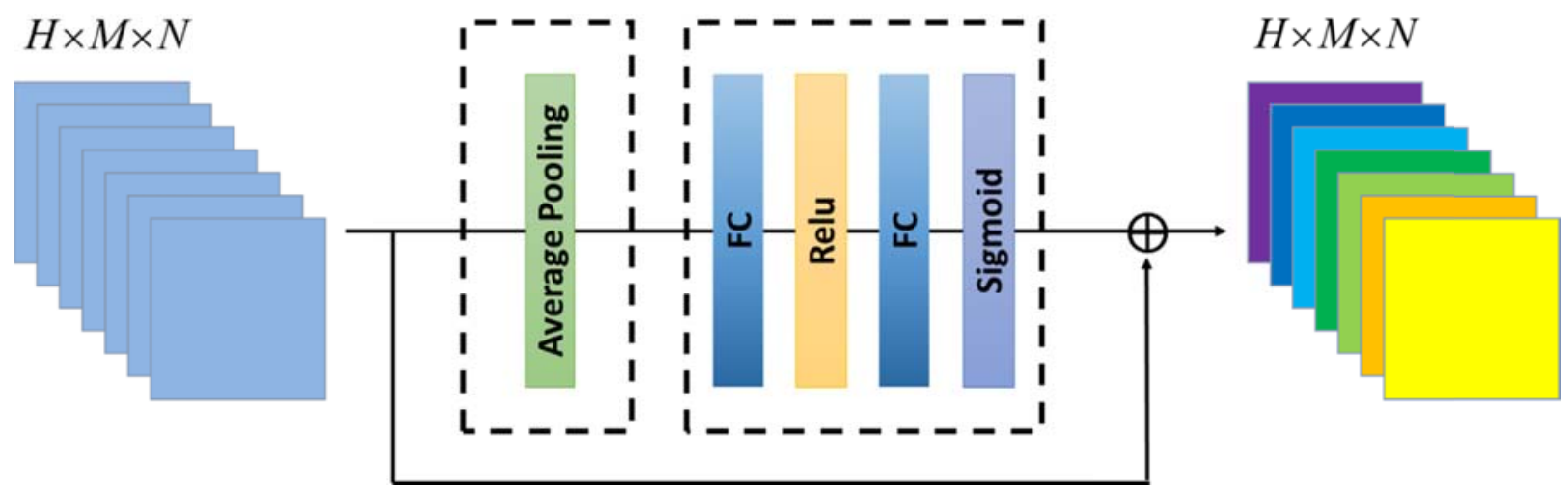

Fig. 5. Channel attention mechanism (CAM)

The squeezing function is similar to the AWMS mechanism, assuming that $\mathrm{X}=\left\{X_{1}, X_{2}, \ldots, X_{C}\right\}$ is the input of CAM and its space size is $M \times N$. After the global average pooling, the global average channel information is $Z=\left\{z_{1}, z_{2}, \ldots, z_{C}\right\}$. The $k$-th element of $Z$ can be expressed as follows:

$$
z_{k}=\operatorname{Ave}\left(X_{k}\right)=\frac{1}{M \times N} \sum_{i=1}^{M} \sum_{j=1}^{N} X_{k}(i, j)
$$

The excitation function consists of two fully connected layers. It is mainly used to generate channel weights for the input feature map; the weights learned to represent the importance of feature channels. Here, the Relu and Sigmoid functions are selected. After obtaining the weight of each feature channel, the weight is multiplied by the corresponding original feature channel. Based on the specific task, the weighted feature maps are obtained. The details are as follows:

$$
\begin{aligned}
& \lambda=\left\{\lambda_{1}, \lambda_{2}, \ldots \lambda_{C}\right\}=\sigma\left(\mathrm{W}_{2} \delta\left(\mathrm{W}_{1} Z\right)\right) \\
& \hat{\mathrm{X}}=\lambda \odot \mathrm{X}=\left\{\hat{X}_{1}, \hat{X}_{2}, \cdots, \hat{X}_{C}\right\}
\end{aligned}
$$


where $\mathrm{W}_{1} \in \mathrm{R}^{\frac{C}{r} \times C}$ and $\mathrm{W}_{2} \in \mathrm{R}^{C \times \frac{C}{r}}$ represent the weights of the two fully connected layers, $r$ is a scaling ratio, $\sigma$ and $\delta$ represent the Sigmoid and Relu functions, respectively. $\lambda=\left\{\lambda_{1}, \lambda_{2}, \ldots \lambda_{C}\right\}$ is the learned weight vector of feature channels. $\odot$ is the channel-wise multiplication, and $\hat{X}$ is the output of CAM. In particular, the $k$-th element in $\hat{X}$ can be expressed as $\hat{X}_{k}=\lambda_{k} \cdot X_{k}$.

\subsection{Network Model Architecture}

Fig. 6 shows the architecture of the proposed network. Input and Output represent the input and output of the network, respectively, where input is a locally normalized $32 \times 32$ image patch. First, the network uses a $3 \times 3$ convolution kernel to extract the detailed features of the input image. Then, the network uses a $9 \times 9$ convolution kernel to extract contour features. During the training process, the quality score of each patch is used as the true label score of its initial image. In the test stage, the true quality score estimation of each image is obtained by averaging the patch prediction score of each image. LIVE and CSIQ databases are used to train a CNN-based NR-IQA network. As shown in Fig. 6, the network consists of three parts: the initial feature extraction, advanced feature extraction, and fully connected network parts. The local normalization method and three main components of the network are described in detail below.

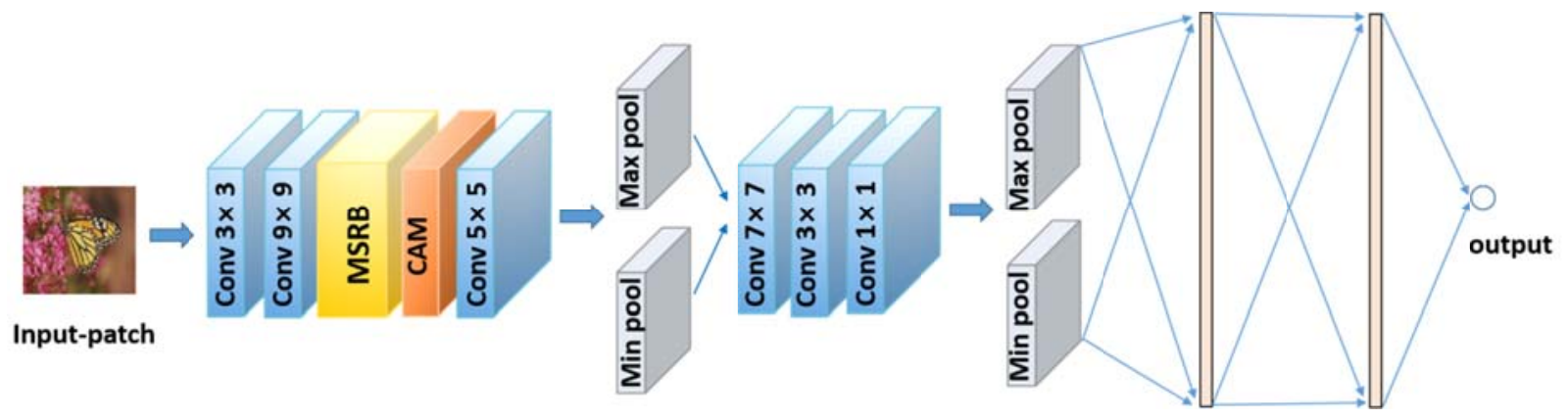

Fig. 6. Model architecture of the proposed network

Local normalization uses a relatively simple normalization method similar to that in [27]. Assuming that the pixel value at the position $(x, y)$ is $I(x, y)$, its normalized value $\hat{I}(x, y)$ can be expressed as follows:

$$
\begin{aligned}
& \hat{I}(x, y)=\frac{I(x, y)-\mu(x, y)}{\sigma(x, y)+\theta} \\
& \mu(x, y)=\frac{1}{P Q} \sum_{p=-P}^{p=P} \sum_{q=-Q}^{q=Q} I(x+p, y+q)
\end{aligned}
$$




$$
\sigma(x, y)=\sqrt{\frac{1}{P Q} \sum_{p=-P}^{p=P} \sum_{q=-Q}^{q=Q}(I(x+p, y+q)-\mu(x, y))^{2}}
$$

where $\theta$ is a small positive number (ensuring that the denominator is not 0 ), and $P Q$ is the total number of image pixels in the local window. In the experiment, this study uses a window with $P=Q=3$, which has a relatively good effect. We obtained that the effect of contrast and brightness of image patch on the learning of network structure can be alleviated by local normalization.

In the initial feature extraction part, the $3 \times 3$ convolution kernel is first used to extract features $p_{1}$ from input. Then, the $9 \times 9$ convolution kernel further extracts contour features and obtains feature information $p_{2}$. The two operations can be described as follows:

$$
\begin{aligned}
& p_{1}=\mathrm{W}_{1}^{3 \times 3} * \text { input }+b_{1} \\
& p_{2}=\mathrm{W}_{2}^{9 \times 9} * p_{1}+b_{2}
\end{aligned}
$$

where $*$ represents the convolution operation, $\mathrm{W}_{1}^{3 \times 3}$ and $b_{1}$ represent the weight matrix and bias of the $3 \times 3$ convolution kernel, respectively. $\mathrm{W}_{2}^{9 \times 9}$ and $b_{2}$ represent the weight matrix and bias of the $9 \times 9$ convolution kernel, respectively. The output $p_{2}$ is the input of the next phase.

The advanced feature extraction part is the core part of the network. First, MSRB is used to extract multi-scale features of $p_{2}$ with AWMS mechanism. Second, CAM is used to learn the correlation between feature channels. Then, the network uses a $5 \times 5$ convolution layer for feature extraction, max pooling, and min pooling for the output features, further using three convolution layers and a parallel pooling layer. Finally, the pooling results are fused into the part of a fully connected network. The advanced feature extraction part is described as follows:

$$
\begin{aligned}
& p_{3}=f\left(p_{2}\right) \\
& p_{4}=g\left(p_{3}\right) \\
& p_{5}=\mathrm{W}_{5}^{5 \times 5} * p_{4}+b_{5} \\
& p_{6}^{1}=\max -\operatorname{pool}\left(p_{5}\right) \\
& p_{6}^{2}=\min -\operatorname{pool}\left(p_{5}\right) \\
& p_{6}=\left[p_{6}^{1}, p_{6}^{2}\right] \\
& p_{7}=\mathrm{W}_{7}^{7 \times 7} * p_{6}+b_{7} \\
& p_{8}=\mathrm{W}_{8}^{3 \times 3} * p_{7}+b_{8} \\
& p_{9}=\mathrm{W}_{9}^{1 \times 1} * p_{8}+b_{9} \\
& p_{10}^{1}=\max -\operatorname{pool}\left(p_{9}\right) \\
& p_{10}^{2}=\min -\operatorname{pool}\left(p_{9}\right) \\
& p_{10}=\left[p_{9}^{1}, p_{9}^{2}\right]
\end{aligned}
$$


where $f(\cdot)$ and $g(\cdot)$ represent the process of using MSRB and CAM, respectively; max-pool( $\cdot)$ and min-pool( $\cdot)$ represent the process of using $2 \times 2$ convolution kernel. Similarly, the output $p_{10}$ of this part is the input of the next stage.

The fully connected network part consists of three fully connected layers. To reduce the complex co-adaptive relationship between neurons, dropout method is used after the first full connection layer, and the ratio is set to 0.5 . This part can be described as follows:

$$
\begin{aligned}
& p_{11}=\max \left(0, \mathrm{~W}_{1} p_{10}\right) \\
& p_{12}=\max \left(0, \mathrm{~W}_{2} p_{11}\right) \\
& \text { output }=p_{13}=\mathrm{W}_{3} p_{12}
\end{aligned}
$$

where $W_{1}, W_{2}$, and $W_{3}$ represent the weight matrices of the three full connection layers with the node number of layers of 100, 800, and 1, respectively. The output represents the final predicted score of a test image.

\subsection{Loss Function}

This study adopts the average absolute error as the optimization objective function. Assume that $x_{n}, y_{n}$ are an input patch and its true score, respectively, and $\theta$ is the parameter of network, then the optimization objective function is defined as follows:

$$
\mathcal{L O S S}=\frac{1}{N} \sum_{i=1}^{N}\left\|\left(f\left(x_{n} ; \theta\right), y_{n}\right)\right\|_{1}
$$

our main objective is to solve the following optimization problem:

$$
\widehat{\theta}=\underset{\theta}{\arg \min } \mathcal{L O S S}=\underset{\theta}{\arg \min }\left(\frac{1}{N} \sum_{i=1}^{N}\left\|\left(f\left(x_{n} ; w\right), y_{n}\right)\right\|_{1}\right)
$$

\section{Experimental results and analysis}

\subsection{Database}

When training the network, patches of $32 \times 32$ size (non-overlapping) are obtained from the initial image and used as the network's input. Compared with taking the initial image as the original input, more training samples can be obtained by partitioning the initial image into patches. Kang et al. [11] pointed out in experiments that the quality score of each patch could be used as the ground truth score of the initial image. Therefore, the average value of the predicted scores for input patches is used in the test stage as the estimate of the quality score of the initial image. This study uses four synthetic databases: LIVE [6], CSIQ [7], TID2008 [8], and TID2013[36]. Besides the synthetic IQA databases, we also validate the proposed model on three in-the-wild IQA databases, including LIVE Challenge [9], KonIQ-10K [10] and SPAQ [38]. Detailed information about the four synthetic databases and three in-the-wild databases is described as follows.

The LIVE database consists of 29 reference images and 779 distorted images. It has five 
types of distortion: JP2K compression (JP2K), JPEG compression (JPEG), white gaussian (WN), gaussian blur (GB), and fast fading (FF). LIVE database provides differential-meansubjective scores (DMOS) for all distorted images, with values in the range $[0,100]$. The closer the DMOS is to 100 , the worse the image quality and the more severe the distortion. Fig. 7 shows the DOMS comparison of the same image with different distortion types in the LIVE dataset.

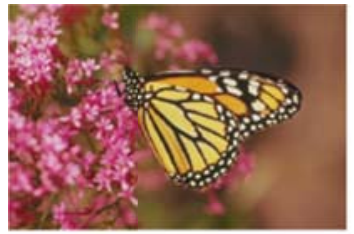

(a) Refimg $\mathrm{DOMS}=0$

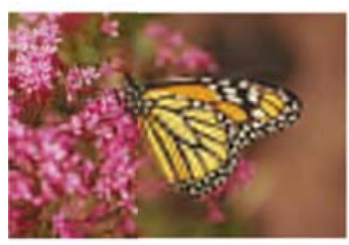

(d) FF DMOS $=65.495$

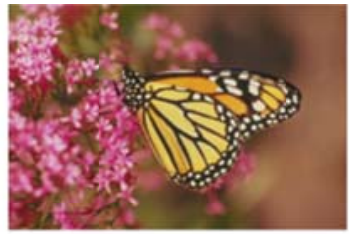

(b) GB DOMS $=21.419$

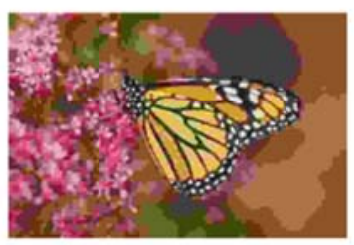

(e) JPEG DOMS $=88.774$

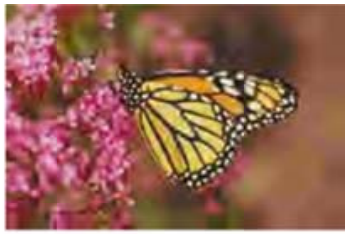

(c) JP2K DMOS $=41.284$

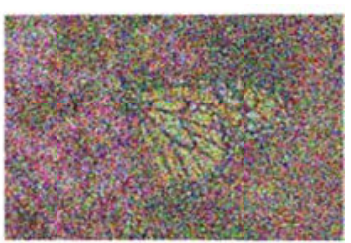

(f) WN DOMS $=94.410$

Fig. 7. Comparison of different distortion types of the LIVE dataset

The CSIQ database consists of 30 reference images and 866 distorted images is a shared database developed by the Computational Perception and Image Lab of Oklahoma State University. There are six types of distortion in the CSIQ database. CSIQ database provides DMOS for all distorted images, with DMOS values in the range [0,1]. The closer the DMOS value is to 1 , the worse the image quality and the more severe the distortion. In this paper, we use a subset of this database with the same distortion types as the LIVE database.

TID2008 was established by the N504 Department of Signal Reception, Transmission, and Processing of the National Aeronautical and Aerospace University of Ukraine. TID2008 consists of 25 reference images and 1700 distorted images. There are 17 types of distortion, such as GB, image noise, JPEG compression, JP2K compression, JPEG transmission error, and JP2K transmission error. TID2008 provides mean-subjective scores (MOS) for all distorted images. The value of MOS is in the range $[0,9]$. Contrary to DMOS, the closer the value is to 0 , the worse the image quality and the more distorted the image.

TID2013 was established by the National Aeronautical and Aerospace University of Ukraine. TID2013 consists of 25 reference images and 3000 distorted images. There are 24 types of distortion, such as GB, JPEG compression and JP2K compression. TID2013 provides mean-subjective scores (MOS) for all distorted images. The value of MOS is in the range $[0$, 9]. In this paper, we use a subset of this database with the same distortion types as the LIVE database. 
LIVE Challenge was established by LIVE Lab of University of Austin, Texas, USA in 2016, containing 1,162 true distorted images. Subjective scores were collected through crowdsourcing with more than 8,100 subject's people. The collected subjective scores exceeded 350,000, and the value range of MOS was [3. 42, 92. 43].

KONIQ-10K (Konstanz Authentic Image Quality Database) was established by University of Konstanz in Germany in 2020, containing 10,073 distorted images. The subjective score was obtained through crowdsourcing with 1,459 annotators and 1.2 million subjective data collected. At the same time, the database also provides image attributes and EXIF (Exchangeable Image File Format) information.

SPAQ (Smartphone Photography Attribute and Quality) was established by Jiangxi University of Finance and Economics in 2020, mainly for the evaluation of mobile phone imaging quality, including 11,125 images collected from 66 different imaging devices. The subjective experiment was carried out in a standard laboratory environment, and the value range of $\operatorname{MOS}$ is $[0,100]$. At the same time, the database provides image attribute information, category information and EXIF information.

\subsection{Evaluation Metrics}

To effectively evaluate the proposed NR-IQA algorithm, it is necessary to evaluate the algorithm's performance. This study uses the Spearman rank correlation coefficient (SROCC), Kendall rank order correlation coefficient (KROCC), Pearson linear correlation coefficient (PLCC) and root mean squared error (RMSE) to evaluate the performance of the proposed IQA algorithm. SROCC, PLCC, KROCC and RMSE were used to measure the monotonicity and accuracy of the NR-IQA results, respectively.

$$
\text { SROCC }=1-\frac{6 \sum_{i=1}^{N} D_{i}^{2}}{N\left(N^{2}-1\right)}
$$

where $D_{i}$ represents the difference between the rankings of the $i-t$ image in the predicted and objective quality scores. The SROCC is in the range of $[-1,1]$. The closer the SROCC is to 1 , the better the performance of the image quality evaluation algorithm.

$$
\text { KROCC }=\frac{n_{c}-n_{d}}{0.5 n(n-1)}
$$

4

where $n_{c}$ is the logarithm of consistent elements in the database and $n_{d}$ is the logarithm of inconsistent elements in the database. The range of KROCC is between $[0,1]$. The closer the KROCC is to 1 , the better the performance of the image quality evaluation algorithm.

$$
\begin{aligned}
P L C C & =\frac{\sum_{i=1}^{N}\left(x_{i}-\bar{x}\right)\left(y_{i}-\bar{y}\right)}{\sqrt{\sum_{i=1}^{N}\left(x_{i}-\bar{x}\right)^{2}} \sqrt{\sum_{i=1}^{N}\left(y_{i}-\bar{y}\right)^{2}}} \\
R M S E & =\sqrt{\frac{1}{n} \sum_{i=1}^{n}\left(x_{i}-y_{i}\right)^{2}}
\end{aligned}
$$


where $x_{i}$ and $y_{i}$ represent the subjective and objective quality score of the $i$-th image, respectively, $\bar{x}$ and $\bar{y}$ represent the average of subjective and objective quality scores, respectively. The range of PLCC is between [0,1]. PLCC is closer to 1 and RMSE is smaller, which proves that the performance of image quality evaluation algorithm is better.

In the experiments, 100 iterations are conducted to train the network. In each networktraining iteration, the training, test, and verification sets are divided according to the ratio of 6:2:2. The proposed network is mainly trained in the Pytorch deep learning framework, which was optimized using the Adam stochastic optimization method. The basic learning rate and weight attenuation were both set to $10 \mathrm{e}-4$, training stops after 100 epochs because there is no further continuous improvement, the data result in the paper is the result of convergence in 100 epochs.

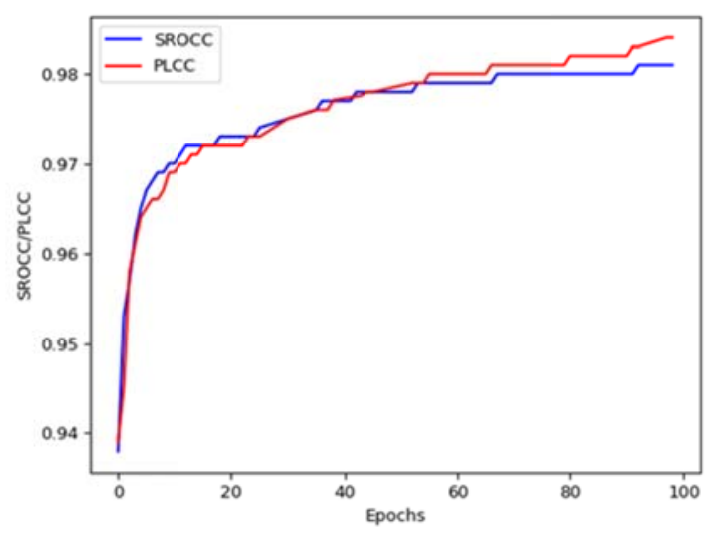

Fig. 8. SROCC and PLCC are under 100 epochs

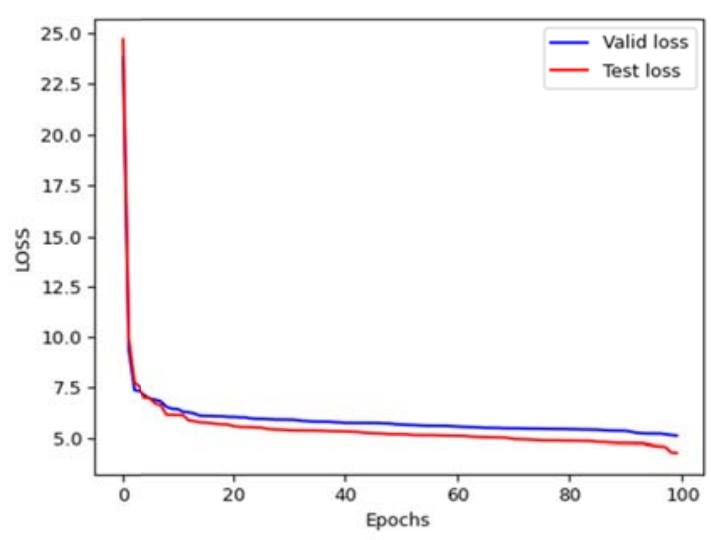

Fig. 9 Loss function graphs are under 100 epochs

\subsection{Evaluation on synthetic databases and in-the-wild databases}

Evaluation on synthetic databases. The distorted images are trained on four synthetic databases. Fig. 8 and Fig.9 show the SROCC, PLCC change and loss charts obtained using 100 epochs of training network on LIVE database, respectively. As shown in the figures, the network converged at about 60 epochs. Table 1 and table 2 compare the most advanced IQA algorithms in four synthetic databases. Specifically, four FR-IQA methods (SSIM [39], PSNR [29] FSIM [30] and VSI [40]) and nine NR-IQA methods (CORNIA [41], BRISQUE [31], CNN [11], CNN++ [42], HOSA [43], RAN4IQA [44], RankIQA [45], dynamic [46], static [46]) were compared in Table 1. Twenty-one methods (NIQE [35], IL-NIQE [57], FRIQUEE [56], BIECON [34], ResNet-ft [58], WaDIQaM-NR [48], MFIQA [59], BMPRI [47], SFA [49], NRVPD [60], ENIQA [61], SGDNet [52], DisNet [53], QUEADI [64], Two-Stream [21], CaHDC [65], DB-CNN [50], HyperIQA [51], UNIQUE [62], HFF [63], MMMNet [66]) were compared in in Table 2. The data results of other methods in the tables are from published 
papers. The best performance results for IQA models in the tables are shown in bold blue, the second-best performance model is shown in red.

Table 1 Performance comparison of state-of-the-art IQA methods based on LIVE, TID2008 and TID2013 databases.

\begin{tabular}{|c|c|c|c|c|c|c|c|c|c|c|c|c|c|}
\hline \multirow{2}{*}{\multicolumn{2}{|c|}{$\begin{array}{l}\text { Databases } \\
\text { Methods }\end{array}$}} & \multicolumn{4}{|c|}{ LIVE } & \multicolumn{4}{|c|}{ TID2008 } & \multicolumn{4}{|c|}{ TID2013 } \\
\hline & & SROCC & KROCC & PLCC & RMSE & SROCC & KROCC & PLCC & RMSE & SROCC & KROCC & PLCC & RMSE \\
\hline \multirow{4}{*}{$\frac{\nwarrow}{\frac{d}{d}}$} & PSNR [39] & 0.872 & 0.690 & 0.903 & 10.145 & 0.579 & 0.422 & 0.592 & 1.080 & 0.646 & 0.471 & 0.006 & 1.230 \\
\hline & SSIM [29] & 0.912 & 0.753 & 0.941 & 7.860 & 0.771 & 0.581 & 0.778 & 0.833 & 0.734 & 0.549 & 0.787 & 0.752 \\
\hline & FSIM [30] & 0.966 & 0.842 & 0.957 & 8.106 & 0.885 & 0.708 & 0.878 & 0.645 & 0.850 & 0.666 & 0.874 & 0.608 \\
\hline & VSI [40] & 0.954 & 0.813 & 0.938 & 9.579 & 0.899 & 0.717 & 0.883 & 0.622 & 0.899 & 0.723 & 0.906 & 0.520 \\
\hline \multirow{10}{*}{$\frac{\nwarrow}{\frac{\nwarrow}{d}}$} & CORNIA [41] & 0.934 & 0.780 & 0.922 & 9.009 & 0.598 & 0.436 & 0.639 & 1.180 & 0.546 & 0.392 & 0.600 & 1.095 \\
\hline & BRISQUE [31] & 0.942 & 0.768 & 0.947 & 8.721 & 0.640 & 0.463 & 0.669 & 1.021 & 0.581 & 0.415 & 0.640 & 0.995 \\
\hline & $\mathrm{CNN}$ [11] & 0.953 & 0.818 & 0.945 & 13.720 & 0.671 & 0.484 & 0.710 & 1.003 & 0.618 & 0.447 & 0.708 & 0.928 \\
\hline & $\mathrm{CNN}++[42]$ & 0.962 & 0.839 & 0.964 & 6.760 & 0.726 & 0.533 & 0.770 & 0.909 & 0.672 & 0.488 & 0.735 & 0.883 \\
\hline & HOSA [43] & 0.946 & 0.808 & 0.939 & 9.253 & 0.743 & 0.545 & 0.759 & 0.950 & 0.712 & 0.526 & 0.756 & 0.880 \\
\hline & RAN4IQA [44] & 0.971 & 0.859 & 0.976 & 6.533 & 0.834 & 0.654 & 0.841 & 0.737 & 0.788 & 0.596 & 0.831 & 0.717 \\
\hline & RankIQA [45] & 0.975 & 0.853 & 0.976 & 11.32 & 0.846 & 0.662 & 0.851 & 1.358 & 0.798 & 0.603 & 0.820 & 1.084 \\
\hline & dynamic [46] & 0.968 & 0.853 & 0.973 & 6.415 & 0.865 & 0.679 & 0.869 & 0.682 & 0.812 & 0.631 & 0.845 & 0.677 \\
\hline & static [46] & 0.977 & 0.875 & 0.981 & 6.130 & 0.862 & 0.681 & 0.864 & 0.663 & 0.820 & 0.640 & 0.842 & 0.699 \\
\hline & MsRCANet & 0.981 & 0.892 & 0.984 & 5.462 & 0.884 & 0.723 & 0.892 & 0.763 & 0.820 & 0.652 & 0.845 & 0.712 \\
\hline
\end{tabular}

Table 2 Performance comparison of state-of-the-art IQA methods based on LIVE and CSIQ databases.

\begin{tabular}{|c|c|c|c|c|c|c|c|c|c|}
\hline \multicolumn{2}{|c|}{ Databases } & \multicolumn{2}{|c|}{ LIVE } & \multicolumn{2}{|c|}{ CSIQ } & \multicolumn{2}{|c|}{ TID2013 } & \multicolumn{2}{|c|}{ Weighted Average } \\
\hline Methods & Source & SROCC & PLCC & SROCC & PLCC & SROCC & PLCC & SROCC & PLCC \\
\hline NIQE [35] & SPL 2012 & 0.912 & 0.901 & 0.883 & 0.901 & 0.811 & 0.824 & 0.869 & 0.875 \\
\hline IL-NIQE [57] & TIP 2015 & 0.902 & 0.908 & 0.821 & 0.865 & 0.521 & 0.648 & 0.748 & 0.807 \\
\hline FRIQUEE [56] & JoV 2017 & 0.940 & 0.944 & 0.835 & 0.874 & 0.680 & 0.753 & 0.818 & 0.857 \\
\hline BIECON [34] & JSTSP 2017 & 0.961 & 0.960 & 0.815 & 0.823 & 0.717 & 0.762 & 0.831 & 0.848 \\
\hline ResNet-ft [58] & SPM 2017 & 0.950 & 0.954 & 0.876 & 0.905 & 0.712 & 0.756 & 0.846 & 0.872 \\
\hline WaDIQaM-NR [48] & TIP 2018 & 0.954 & 0.954 & 0.963 & 0.852 & 0.844 & 0.787 & 0.761 & 0.864 \\
\hline MFIQA [59] & ICIP 2018 & 0.970 & 0.972 & 0.844 & 0.880 & 0.825 & 0.850 & 0.880 & 0.901 \\
\hline BMPRI [47] & ТВC 2018 & 0.931 & 0.933 & 0.909 & 0.933 & 0.928 & 0.947 & 0.923 & 0.938 \\
\hline SFA [49] & TMM 2018 & 0.883 & 0.895 & 0.796 & 0.818 & 0.818 & 0.954 & 0.832 & 0.889 \\
\hline NRVPD [60] & Inf.2019 & 0.943 & 0.947 & 0.840 & 0.889 & 0.683 & 0.768 & 0.822 & 0.868 \\
\hline ENIQA [61] & JIVP 2019 & 0.938 & 0.944 & 0.851 & 0.873 & 0.702 & 0.750 & 0.830 & 0.856 \\
\hline SGDNet [52] & MM 2019 & 0.965 & 0.969 & 0.883 & 0.903 & 0.843 & 0.861 & 0.897 & 0.911 \\
\hline DisNet [53] & SPL 2019 & 0.950 & 0.950 & 0.880 & 0.910 & 0.790 & 0.820 & 0.873 & 0.893 \\
\hline QUEADI [64] & SIViP 2020 & 0.844 & 0.831 & 0.641 & 0.698 & 0.533 & 0.615 & 0.673 & 0.715 \\
\hline Two-Stream [21] & IET IP 2020 & 0.942 & 0.944 & 0.862 & 0.886 & 0.736 & 0.786 & 0.847 & 0.872 \\
\hline $\mathrm{CaHDC}[65]$ & TIP 2020 & 0.965 & 0.964 & 0.903 & 0.914 & 0.862 & 0.885 & 0.910 & 0.921 \\
\hline $\mathrm{DB}-\mathrm{CNN}[50]$ & TCSVT 2020 & 0.968 & 0.971 & 0.946 & 0.959 & 0.816 & 0.865 & 0.910 & 0.932 \\
\hline HyperIQA [51] & CVPR 2020 & 0.962 & 0.966 & 0.923 & 0.942 & - & - & - & - \\
\hline UNIQUE [62] & TIP 2021 & 0.969 & 0.968 & 0.902 & 0.927 & - & - & - & - \\
\hline HFF [63] & TMM 2021 & 0.964 & - & 0.919 & - & - & - & - & - \\
\hline MMMNet [66] & TCSVT 2021 & 0.970 & 0.970 & 0.924 & 0.937 & 0.832 & 0.853 & 0.909 & 0.920 \\
\hline MsRCANet & & 0.981 & 0.984 & 0.955 & 0.966 & 0.830 & 0.855 & 0.919 & 0.932 \\
\hline
\end{tabular}

Table 1 lists the results of SROCC, PLCC, KROCC and RMSE on the synthetic databases LIVE, TID2008 and TID2013. It can be seen from Table 1 that our algorithm is superior to all 
of the compared FR-IQA and NR-IQA methods. Table 2 lists the results of SROCC and PLCC on the synthetic databases LIVE, CSIQ, TID2013 and average values of the three databases. As can be seen from the Table 2, compared with other most advanced models, except TID2013 database, the network performance proposed is the best and at the same time the most competitive model. After averaging the SROCC and PLCC of the three synthetic databases, it can be seen that the proposed model has the best performance, which indicates that this model has strong generalization ability and is superior to other NR-IQA algorithms without sacrificing too many resources. In order to better show that the network we proposed has good generalization ability, we tested the proposed network and other algorithms (PSNR [29], FSIM [30], BRISQUE [31], GM-log [32], CNN [11], BIECON [34]) in LIVE, CSIQ and TID2008 databases. The results show that the proposed network achieves good results for several kinds of distorted data. The experimental results are shown in Table 3. The results of the proposed network on SROCC are better than those of other networks, indicating that the proposed network is relatively effective in evaluating these distorted images.

Table 3 Performance comparison of SROCC under different types of distortion in LIVE, CSIQ and TID2008 databases.

\begin{tabular}{|c|c|ccccccc|}
\hline \multirow{2}{*}{ Databases } & Type & PSNR [29] & FSIM [30] & BRISQUE [31] & GM-LOG [32] & CNN [11] & BIECON [34] & MsRCANet \\
\hline \multirow{6}{*}{ LIVE } & JP2K & 0.870 & 0.970 & 0.914 & 0.928 & 0.952 & 0.952 & $\mathbf{0 . 9 7 5}$ \\
& JPEG & 0.885 & 0.981 & 0.965 & 0.966 & 0.977 & 0.974 & $\mathbf{0 . 9 8 5}$ \\
& WN & 0.942 & 0.967 & 0.979 & 0.983 & 0.978 & 0.980 & $\mathbf{0 . 9 9 2}$ \\
& GB & 0.763 & 0.972 & 0.951 & 0.939 & 0.962 & 0.956 & $\mathbf{0 . 9 8 6}$ \\
& FF & 0.874 & 0.949 & 0.877 & 0.966 & 0.956 & 0.923 & $\mathbf{0 . 9 6 7}$ \\
\hline \multirow{5}{*}{ CSIQ } & JP2K & 0.894 & 0.984 & 0.894 & 0.917 & 0.946 & 0.948 & $\mathbf{0 . 9 4 9}$ \\
& JPEG & 0.854 & 0.950 & 0.925 & 0.934 & 0.939 & 0.938 & $\mathbf{0 . 9 6 9}$ \\
& WN & 0.933 & 0.921 & 0.931 & 0.942 & 0.948 & 0.955 & $\mathbf{0 . 9 6 7}$ \\
& GB & 0.939 & 0.943 & 0.914 & 0.907 & 0.956 & 0.963 & $\mathbf{0 . 9 6 6}$ \\
\hline \multirow{5}{*}{ TID2008 } & JP2K & 0.894 & 0.974 & 0.901 & 0.927 & 0.940 & 0.946 & $\mathbf{0 . 9 5 2}$ \\
& JPEG & 0.858 & 0.955 & 0.911 & 0.934 & 0.949 & 0.947 & $\mathbf{0 . 9 5 1}$ \\
& WN & 0.933 & 0.911 & 0.970 & 0.907 & 0.899 & 0.913 & $\mathbf{0 . 9 5 6}$ \\
& GB & 0.938 & 0.972 & 0.923 & 0.881 & 0.959 & 0.967 & $\mathbf{0 . 9 6 9}$ \\
\hline
\end{tabular}

Evaluation on in-the-wild databases. The distorted images were trained on three in-thewild IQA databases. Table 4 compares the performance results of the proposed algorithm with six popular IQA algorithms on three in-the-wild databases. Specifically, these algorithms include QAC [33], NIQE [35], BRISQUE [31] and BMPRI [47], CNN [11], WaDIQaM-NR [48]. The data results of other six algorithms in the tables are from published papers. The best performance is highlighted in each column. It can be seen from the Table 4 that although the performance of our proposed algorithm on the three In-the-wild IQA databases is not always the best, it also achieved better performance results, which indicates that our model proposed has good generalization ability on in-the-wild IQA databases. The best performance results for IQA models in the table are shown in bold blue, the second-best performance model is shown in red. 
Table 4 Performance of eleven state-of-the-art methods and the proposed model on three in-the-wild IQA databases.

\begin{tabular}{|l|l|cccccccc|}
\hline \multicolumn{2}{|c|}{ Databases } & QAC [33] & NIQE [35] & BRISQUE [31] & BMPRI [47] & CNN [11] & WaDIQaM-NR [48] & MsRCANet \\
\hline LIVE & SROCC & 0.069 & 0.454 & 0.601 & 0.487 & 0.627 & 0.671 & $\mathbf{0 . 7 7 9}$ \\
Challenge & PLCC & 0.014 & 0.468 & 0.621 & 0.523 & 0.601 & 0.680 & $\mathbf{0 . 8 0 2}$ \\
\hline \multirow{2}{*}{ KonIQ10k } & SROCC & 0.343 & 0.526 & 0.715 & 0.658 & 0.685 & 0.710 & $\mathbf{0 . 7 2 4}$ \\
& PLCC & 0.296 & 0.475 & 0.702 & 0.655 & 0.684 & 0.738 & 0.731 \\
\hline \multirow{2}{*}{ SPQA } & SROCC & 0.047 & 0.697 & 0.802 & 0.750 & 0.796 & 0.837 & $\mathbf{0 . 8 3 9}$ \\
& PLCC & 0.107 & 0.685 & 0.806 & 0.754 & 0.799 & $\mathbf{0 . 8 4 5}$ & 0.843 \\
Weighted & SROCC & 0.153 & 0.559 & 0.706 & 0.632 & 0.703 & 0.739 & $\mathbf{0 . 7 8 1}$ \\
Average & PLCC & 0.139 & 0.543 & 0.710 & 0.644 & 0.695 & 0.754 & $\mathbf{0 . 7 9 2}$ \\
\hline
\end{tabular}

\subsection{Cross-Database Evaluations}

To test the generalization ability of the proposed network model on different databases, this section trains the proposed network model on three synthetic databases LIVE, CSIQ, TID2013 and one in-the-wild IQA database LIVE Challenge, and test network performance on the corresponding three databases. The proposed mode is compared with BRISQUE [31], GMLOG [32], FRIQUEE [56], CORNIA [41], HOSA [43], WaDIQaM-NR [48], DB-CNN [50], MMMNet [66]. The obtained SROCC results are shown in Table 5. The results of other algorithms in the Table 5 are obtained from published papers. As can be seen in Table 5, under such challenging experiments, the generalization ability of the proposed model is better than the most advanced NR-IQA models, which proves the effectiveness of the proposed method.

Table 5 SROCC results in the cross-database setting.

\begin{tabular}{|c|c|c|c|c|c|c|c|c|c|c|c|c|}
\hline \multirow{2}{*}{$\begin{array}{l}\text { Training } \\
\text { Testing }\end{array}$} & \multicolumn{3}{|c|}{ LIVE } & \multicolumn{3}{|c|}{ CSIQ } & \multicolumn{3}{|c|}{ TID2013 } & \multicolumn{3}{|c|}{ LIVE Challenge } \\
\hline & CSIQ & TID2013 & $\begin{array}{c}\text { LIVE } \\
\text { Challenge }\end{array}$ & LIVE & TID2013 & $\begin{array}{c}\text { LIVE } \\
\text { Challenge }\end{array}$ & LIVE & CSIQ & $\begin{array}{c}\text { LIVE } \\
\text { Challenge }\end{array}$ & LIVE & CSIQ & TID2013 \\
\hline BRISQUE [31] & 0.562 & 0.358 & 0.337 & 0.847 & 0.454 & 0.131 & 0.790 & 0.590 & 0.254 & 0.238 & 0.241 & 0.280 \\
\hline GM-LOG [32] & 0.621 & 0.344 & 0.226 & 0.797 & 0.328 & 0.183 & 0.873 & 0.605 & 0.112 & 0.059 & 0.109 & 0.058 \\
\hline FRIQUEE [56] & 0.722 & 0.461 & 0.411 & 0.879 & 0.463 & 0.264 & 0.755 & 0.635 & 0.181 & 0.644 & 0.592 & 0.424 \\
\hline CORNIA [41] & 0.649 & 0.360 & 0.443 & 0.853 & 0.312 & 0.393 & 0.846 & 0.672 & 0.293 & 0.588 & 0.446 & 0.403 \\
\hline HOSA [43] & 0.594 & 0.361 & 0.463 & 0.733 & 0.329 & 0.291 & 0.846 & 0.612 & 0.319 & 0.537 & 0.336 & 0.399 \\
\hline WaDIQaM-NR [48] & 0.704 & 0.462 & - & - & - & - & - & 0.733 & - & - & - & - \\
\hline DB-CNN [50] & 0.758 & 0.524 & 0.567 & 0.877 & 0.540 & 0.452 & 0.891 & 0.807 & 0.457 & 0.746 & 0.697 & 0.424 \\
\hline MMMNet [66] & 0.793 & 0.546 & 0.502 & 0.890 & 0.522 & 0.406 & 0.853 & 0.702 & 0.348 & 0.528 & 0.518 & 0.398 \\
\hline MsRCANet & 0.921 & 0.663 & 0.952 & 0.934 & 0.780 & 0.824 & 0.962 & 0.942 & 0.691 & 0.953 & 0.959 & 0.626 \\
\hline
\end{tabular}

\subsection{Ablation Study}

In this section, a comparative experiment is first conducted on the size of the input patch. According to the results in Table 6, the best evaluation effect is achieved when the patch size 
was $32 \times 32$. Therefore, the patch size was set as $32 \times 32$ in our experiments. Additionally, to verify the effectiveness of the proposed MSRB and CAM, a comparative experiment was conducted on the LIVE database to see whether MSRB and CAM were used (see Table 6). As shown in Table 7, the model with feature extraction block MSRB and CAM is superior to that without MSRB and CAM. Therefore, it can be concluded that multi-scale feature extraction and CAM improve the neural networks' evaluation effect on the LIVE database.

Table 6 SROCC and PLCC are in different patch size on LIVE database.

\begin{tabular}{|cccc|}
\hline Size & $16 \times 16$ & $32 \times 32$ & $64 \times 64$ \\
\hline SROCC & 0.956 & $\mathbf{0 . 9 8 1}$ & 0.951 \\
PLCC & 0.961 & $\mathbf{0 . 9 8 4}$ & 0.947 \\
\hline
\end{tabular}

Table 7 SROCC and PLCC are in different components on LIVE database.

\begin{tabular}{|cccc|}
\hline MSRB & CAM & SROCC & PLCC \\
\hline$\times$ & $\times$ & 0.952 & 0.948 \\
$\sqrt{ }$ & $\times$ & 0.960 & 0.963 \\
$\times$ & $\sqrt{ }$ & 0.959 & 0.955 \\
$\sqrt{ }$ & $\sqrt{ }$ & $\mathbf{0 . 9 8 1}$ & $\mathbf{0 . 9 8 4}$ \\
\hline
\end{tabular}

\section{Conclusion}

In this study, a novel no-reference quality assessment model based on a deep neural network (MsRCANet) is proposed. This proposed model extracts contour features and processes detailed features to learn better and predict image quality features, making the IQA more accurate. In the MsRCANet, MSRB with three parallel branches is first used to extract distorted images. In MSRB, residual learning with an AWMS is used to redesign the input mapping instead of directly adding the two results of the residual learning. Since different channels process feature information differently, the next step is to use CAM to learn the dependencies between channels. In this way, the corresponding weight values of different paths are deduced from the mechanism.

The proposed method's performance is analyzed through experiments. The MsRCANet is superior to most advanced NR-IQA methods and has a better performance of image quality evaluation. It is highly competitive with some FR-IQA methods and existing NR-IQA methods. Although the MsRCANet has achieved good performance in image quality evaluation, there are still some areas to be improved. For example, how to use other more efficient methods to improve the patch prediction score as a prediction score for the entire picture, and how can we improve the performance of in-the-wild IQA databases better? In future studies, we will focus on studying more effective methods to realize IQA. Meanwhile, we will focus on NR-IQA and explore more effective and practical models in more fields.

\section{ACKNOWLEDGMENTS}


This work was supported by National Natural Science Foundation of China under Grants 61976027, 61572082, Liaoning Revitalization Talents Program (XLYC2008002).

\section{Compliance with Ethical Standards}

The authors declare that they have no conflict of interest. All procedures performed in studies involving human participants were in accordance with the ethical standards of the institutional and/or national research committee and with the 1964 Helsinki declaration and its later amendments or comparable ethical standards. This article does not contain any studies with animals performed by any of the authors. Informed consent was obtained from all individual participants included in the study.

\section{Authorship contributions}

All authors contributed to the study conception and network design. Algorithm preparation, data collection and analysis were performed by Changzhong Wang, Xiang Lv and Xiaodong Fan. The first draft of the manuscript was written by Xiang Lv and all authors commented on previous versions of the manuscript. All authors read and approved the final manuscript.

\section{References}

[1] Hong, Hao, et al. Image detail enhancement with spatially guided filters. Signal Processing: The Official Publication of the European Association for Signal Processing (EURASIP), 120 (2016) 789-796.

[2] S. Hao, Y. Guo, Z. Wei, et al. Lightness-aware contrast enhancement for images with different illumination conditions. Multimedia Tools and Applications, 78 (2019) 3817-3830.

[3] A. Holzinger. From Machine Learning to Explainable AI. World Symposium on Digital Intelligence for Systems and Machines (DISA), 2018, pp. 55-66.

[4] A。 Holzinger. Explainable AI and Multi-Modal Causability in Medicine. i-com, 19 (3) (2021) 171-179.

[5] X. Jin, L. Wu, X. Li, et al. ILGNet: inception modules with connected local and global features for efficient image aesthetic quality classification using domain adaptation. IET Computer Vision, (2016) 1-6.

[6] H. Sheikh, M. Sabir, A. Bovik. A Statistical Evaluation of Recent Full Reference Image Quality Assessment Algorithms. IEEE Transactions on Image Processing, 15 (11) (2006) 3440-3451.

[7] C. Larson, M. Chandler. Most apparent distortion: full-reference image quality assessment and the role of strategy. Journal of Electronic Imaging, 19 (1) (2010) 011006.

[8] N. Ponomarenko, V. Lukin, A. Zelensky, et al. TID2008-a database for evaluation of full-reference visual quality assessment metrics. Advances of Modern Radio electronics, 10 (4) (2009) 30-45.

[9] G. Deepti and C. Alan. Massive online crowdsourced study of subjective and objective picture quality. IEEE Transactions on Image Processing, 25 (1) (2015) 372-387.

[10]H. Vlad, L. Hanhe, et al. KonIQ-10k: An Ecologically Valid Database for Deep Learning of Blind Image Quality Assessment. IEEE Transactions on Image Processing, 29 (2020) 4041-4056.

[11]L. Kang, P. Ye, Y. Li, and D. Doermann. Convolutional neural networks for no-reference image quality assessment. in Proc. IEEE Conf. Comput. Vis. Pattern Recognit., Jun. 2014, pp. 1733-1740.

[12]Y. Li, L.-M. Po, L. Feng, and F. Yuan. No-reference image quality assessment with deep convolutional neural networks. in Proc. IEEE Int. Conf. Digit. Signal Process. (DSP), 2016, pp. 685-689.

[13]P. P. Dash, A. Wong, and A. Mishra, VeNICE: A very deep neural network approach to no-reference image assessment. in Proc. IEEE Int. Conf. Ind. Technol. (ICIT), 2017, pp. 1091-1096.

[14]C. Sun, H. Li, and W. Li. No-reference image quality assessment based on global and local content perception. in Proc. Vis. Commun. Image Process., 2016, pp. 1-4.

[15]S. Bosse, D. Maniry, T. Wiegand, and W. Samek. A deep neural network for image quality assessment. in Proc. IEEE Int. Conf. Image Process., Phoenix, AZ, USA, Sep. 2016, pp. 3773-3777. 
[16]C. Pan, Y. Xu, Y. Yan, K. Gu, and X. Yang. Exploiting neural models for no-reference image quality assessment. in Proc. Vis. Commun. Image Process., 2016, pp. 1-4.

[17]L. Zuo, H. Wang, and J. Fu. Screen content image quality assessment via convolutional neural network. in Proc. IEEE Int. Conf. Image Process. (ICIP), 2016, pp. 2082-2086.

[18]Z. Cheng, M. Takeuchi, and J. Katto. A pre-saliency map based blind image quality assessment via convolutional neural networks. in Proc. IEEE Int. Symp. Multimedia (ISM), 2017, pp. 77-82.

[19]J. Kim, S. Lee. Fully Deep Blind Image Quality Predictor. Selected Topics in Signal Processing, IEEE Journal of, 11 (1) (2017) 206-220.

[20]K. Lin, G. Wang. Hallucinated-IQA: No-Reference Image Quality Assessment via Adversarial Learning. in Proc. IEEE Conf. Comput. Vis. Pattern Recognit., 2018.

[21]Q. Yang, D. Gong, Y. Zhang. Two-Stream Convolutional Networks for Blind Image Quality Assessment. IEEE transactions on image processing, 28 (5) (2019) 2200.

[22]K. He, X. Zhang, S. Ren, J. Sun. Deep residual learning for image recognition, in Proc. IEEE Conf. Comput. Vis. Pattern Recognit., 2016, pp. 770-778.

[23]Y. Zhang, Y. Tian, Y. Kong, B. Zhong, Y. Fu, Residual dense network for image super-resolution. in Proc. IEEE Conf. Comput. Vis. Pattern Recognit., 2018, pp. 2472-2481.

[24]J. Hu, L. Shen, G. Sun, Squeeze-and-excitation networks. in Proc. IEEE Conf. Comput. Vis., 2018, pp. $7132-7141$.

[25]Y. Zhang, K. Li, et al. Image Super-Resolution Using Very Deep Residual Channel Attention Networks, 2018.

[26]J. Hyoungho, L. Ryong, L. Sanghwan, et al. Residual Convolutional Neural Network Revisited with Active Weighted Mapping, 2018.

[27]W. Zhang, C. Qu, L. Ma, et al. Learning structure of stereoscopic image for no-reference quality assessment with convolutional neural network. Pattern Recognition, (2016) 176-187.

[28]P. Ye, J. Kumar, L. Kang, and D. Doermann. Real-time no-reference image quality assessment based on filter learning. in Proc. IEEE Conf. Comput. Vis. Pattern Recognit., 2013, pp. 987-994.

[29]Z. Wang, A.C. Bovik, A universal image quality index. IEEE Sign. Process. Lett, 9 (3) (2002) 81-84.

[30]L. Zhang, Mou X, et al. FSIM: a feature similarity index for image quality assessment. IEEE Trans Image Process, 20 (8) (2011) 2378-2386.

[31]M. Anish, K. Anush, C. Alan Bovik. No-reference image quality assessment in the spatial domain. IEEE Trans Image Process, 12 (2012) 4695-4708.

[32]W. Xue, X. Mou, L. Zhang, et al. Blind image quality assessment using joint statistics of gradient magnitude and Laplacian features. IEEE Trans Image Process, 23 (11) (2014) 4850-4862.

[33]W. Xue, L.Zhang, X. Mou. Learning without human scores for blind image quality assessment. in Proc. IEEE Conf. Comput. Vis. Pattern Recognit., 2013, pp. 995-1002.

[34]Kim J, Lee S (2017) Fully deep blind image quality predictor. IEEE J Select Topics Signal Process 11(1):206-220.

[35]Anish Mittal, Rajiv Soundararajan, and Alan C Bovik. 2012. Making a completely blind" image quality analyzer. IEEE Signal Processing Letters, 20, 3 (2012), 209-212.

[36]N. Ponomarenko, et al. Image database TID2013: Peculiarities, results and perspectives. Signal Process. Image Commun., (2015) 57-77.

[37]P. Chen, Y. Niu, D. Huang. No-Reference Image Quality Assessment Based on Multi-scale Convolutional Neural Networks. 2019.

[38]Y. Fang, H. Zhu, Y. Zeng, et al. Perceptual Quality Assessment of Smartphone Photography. in Proc. IEEE Conf. Comput. Vis. Pattern Recognit., 2020, pp. 3677-3686.

[39]Z. Wang, A. Bovik, H. Sheikh, et al. Image quality assessment: From error visibility to structural similarity. IEEE Trans Image Process., (2004) 600-612.

[40]L. Zhang, Y. Shen, and H. Li, "VSI: A visual saliency-induced index for perceptual image quality assessment," IEEE Trans. Image Process., (2014) 4270-4281.

[41]P. Ye, J. Kumar, L. Kang, D. Doermann. Unsupervised featurelearning framework for no-reference image quality assessment. in Proc. IEEE Conf. Comput. Vis. Pattern Recognit., 2012, pp. 1098-1105.

[42]L. Kang, P. Ye, Y. Li, D. Doermann. Simultaneous estimation of image quality and distortion via multi-task convolutional neural networks. in Proc. IEEE Int. Conf. Image Process, 2015, pp. 2791-2795. 
[43]J. Xu, P. Ye, Q. Li, H. Du, Y. Liu, D. Doermann. Blind image quality assessment based on high order statistics aggregation. IEEE Trans. Image Process., 2016. pp. 4444-4457.

[44]H. Ren, D. Chen, Y. Wang. RAN4IQA: Restorative adversarial nets for no-reference image quality assessment. in Proc. 32nd AAAI Conf. Artif. Intell., 2018, pp. 7308-7314.

[45]X. Liu, A. Bagdanov. RankIQA: Learning from rankings for no-reference image quality assessment. 2017.

[46]D. Chen, Y. Wang, W. Gao. No-Reference Image Quality Assessment: An Attention Driven Approach. IEEE Transactions on Image Processing, 29 (99) (2020) 6496-6506.

[47]X. Min, G. Zhai, K. Gu, Y. Liu, et al. Blind Image Quality Estimation via Distortion Aggravation. IEEE Transactions on Broadcasting, 64 (2) (2018) 508-517.

[48]S. Bosse, D. Maniry, K. Müller, et al. Deep neural networks for no-reference and full-reference image quality assessment. IEEE Trans. Image Process, 27 (1) (2017) 206-219.

[49]D. Li, T. Jiang, W. Lin, and M. Jiang. Which has better visual quality: The clear blue sky or a blurry animal? IEEE Transactions on Multimedia, 21 (5) (2018) 1221-1234.

[50]Wei. Zhang, Ke. Ma, J.Yan, et al. Blind Image Quality Assessment Using a Deep Bilinear Convolutional Neural Network. IEEE Transactions on Circuits and Systems for Video Technology, 30 (1) (2020) 36-47.

[51]S. Su, Q. Yan, Y. Zhu, et al. Blindly Assess Image Quality in the Wild Guided by a Self-Adaptive Hyper Network. in Proc. IEEE Conf. Comput. Vis. Pattern Recognit., 2020, pp. 3667-3676.

[52]S. Yang, Q. Jiang, W. Lin, and Y. Wang. SGDNet: An end-to-end saliency-guided deep neural network for no-reference image quality assessment, in Proc. ACM International Conference on Multimedia Association for Computing Machinery, (2019) 1383-1391.

[53]S. Dendi, C. Dev, et al. Generating image distortion maps using convolutional autoencoders with application to no reference image quality assessment, IEEE Signal Processing Letters, 26 (1) (2019) 89-93.

[54]Li, F. Fang, K. Mei, G. Zhang. Multi-scale residual network for image super-resolution, in Proc. Eur. Conf. Comput. Vis., (2018) 527-542.

[55]W. Sun, T. Wang, X. Min, et al. Deep Learning Based Full-Reference and No-Reference Quality Assessment Models for Compressed UGC Videos." IEEE International Conference on Multimedia \& Expo Workshops (ICMEW) IEEE, 2021.

[56]D. Ghadiyaram and A. C. Bovik. Perceptual quality prediction on authentically distorted images using a bag of features approach, Journal of Vision, (2017) 32-32.

[57]L. Zhang, L. Zhang, and A. C. Bovik. A feature-enriched completely blind image quality evaluator, IEEE Trans. Image Process., 24 (8) (2015)2579-2591.

[58]J. Kim, H. Zeng, D. Ghadiyaram, S. Lee, L. Zhang, and A. C.Bovik. Deep convolutional neural models for picture-quality prediction: Challenges and solutions to data-driven image quality assessment, IEEE Signal Proc. Mag., 34 (6) (2017) 130 141.

[59]J. Kim, A. Nguyen, S. Ahn, C. Luo and S. Lee, "Multiple level feature-based universal blind image quality assessment model," in Proc ICIP, 2018, pp. 291-295.

[60]J. Wu, M. Zhang, L. Li, W. Dong, G. W. Lin. No-Reference Image Quality Assessment with Visual Pattern Degradation, Inf. Sci., (2019) 487-500.

[61]X. Chen, Q. Zhang, M. Lin, et al. No-reference color image quality assessment: From entropy to perceptual quality, J Image Video Proc,2019.

[62]W. Zhang, K. Ma, G. Zhai, et al. Uncertainty-Aware Blind Image Quality Assessment in the Laboratory and Wild, IEEE Transactions on Image Processing, (2021) 3474-3486.

[63]W. Sun, X. Min, G. Zhai, \& S. Ma, Blind Quality Assessment for in-the-Wild Images via Hierarchical Feature Fusion and Iterative Mixed Database Training, 2021.

[64]Rajchel, Mariusz, et al. No-reference image quality assessment of authentically distorted images with global and local statistics, Signal, Image and Video Processing, (SIViP),2020.

[65]J. Wu, J. Ma, F. Liang, W. Dong, G. Shi and W. Lin. End-to-End Blind Image Quality Prediction With Cascaded Deep Neural Network, IEEE Trans. Image Process., (2020) 7414-7426.

[66]F. Li, Y. Zhang, et al. MMMNet: An End-to-End Multi-task Deep Convolution Neural Network with Multi-scale and Multi-hierarchy Fusion for Blind Image Quality Assessment. IEEE Transactions on Circuits and Systems for Video Technology, 2021. 